\title{
Article
}

\section{Chip-Based Digital PCR improves the detection of low-rate PIK3CA mutations in Breast Cancer Patients}

\author{
Stefano Giannoni-Luza ${ }^{1}$, Oscar Acosta Conchucos ${ }^{1,2}$, Alexis Germán Murillo Carrasco ${ }^{1}$, Pierina Danos ${ }^{1}$, José Ma- \\ nuel Cotrina Concha ${ }^{3}$, Henry Guerra Miller ${ }^{3}$, Joseph Arturo Pinto Oblitas ${ }^{4}$, Alfredo Aguilar Cartagena ${ }^{4}$, Jhahaira \\ Araujo Soria ${ }^{4}$, Ricardo Fujita ${ }^{1}$ and José Luis Buleje Sono ${ }^{1,4^{*}}$
}

\author{
1. Centro de Genética y Biología Molecular, Universidad de San Martin de Porres. Lima-Peru. \\ 2. Facultad de Farmacia y Bioquímica, Universidad Nacional Mayor de San Marcos, Lima, Perú \\ 3. Instituto Nacional de Enfermedades Neoclásicas (INEN). Lima-Peru. \\ 4. Oncosalud-Auna. Lima-Peru. \\ 5. Escuela Profesional de Medicina Humana, Filial Ica, Universidad Privada San Juan Bautista, Carretera Pan- \\ americana Sur Km 300, Ica, 11004, Peru. \\ * Correspondence: jbulejes@gmail.com. Phone: +51 985225663.
}

Simple Summary: PIK3CA mutations are frequent mutations in breast cancer and are considered as potential predictive and prognosis factors. Novel platforms for gene analysis as digital PCR (dPCR) are emerging as a potential replacement for traditional Sanger sequencing. However, there are still few studies on dPCR. Thus, this cross-sectional study aimed to assess the sensibility of dPCR to detect and quantify PIK3CA mutations in breast cancer patients and compare its performance with Sanger sequencing. Our findings describe $\mathrm{APCR}$ as a technique able to detect low-rate PIK3CA mutations. It represents tumor subpopulations not usually detected by Sanger sequencing, which could induce treatment changes at clinical routine.

\begin{abstract}
PIK3CA is a gene usually mutated in breast cancer and has an important role in tumor progression and treatment. Therefore, there is required a technique to detect low-rate PIK3CA mutations improving the clinical conduct. This study aimed to compare chip-based dPCR and Sanger sequencing to detect PIK3CA mutations in breast cancer patients. Fifty-seven tumor samples from breast cancer patients were collected and analyzed by Sanger sequencing and APCR for PIK3CA mutations (E545K, H1047R, and H1047L). Digital PCR sensitivity, specificity, and overall performance were estimated by contingency tables, receptor operator characteristic (ROC), and area under the curve (AUC). Sanger sequencing identified PIK3CA mutations in six patients (10.5\%), two with H1047R, and four with E545K. Digital PCR confirmed those mutations and identified 19 additional patients with at least one mutation. Comparison between dPCR and Sanger sequencing showed a sensitivity of $100 \%$ (95\% CI 53-100\%), and a specificity of $84.2 \%$ (95\% CI $83-84.2 \%$ ). Besides, H1047R mutation showed a significant association with breast cancer phenotype $(p=0.019)$ and lymphatic node infiltration ( $p=0.046$ ). Digital PCR showed a high sensitivity to detect mutations in tumor samples and it might be capable to detect low-rate mutations and tumor subpopulations not detected by Sanger sequencing.
\end{abstract}

Keywords: Breast cancer; PIK3CA; digital PCR; Sanger sequencing

\section{Introduction}

PIK3CA mutations are common in breast cancer as described by The Cancer Genetic Atlas (TGCA) with a frequency of $36 \%$ [1]. Although this gene has 23 exons, their mutations are clustered in two hot spots in exon 09 (E542K and E545K) and exon 20 (H1047R and H1047L) [2]. The PIK3CA gene is located in chromosome 3q26.3 and encode the p110 $\alpha$ isoform, part of the phosphatidilinositol 3-kinase (PI3K)/AKT/mTOR pathway [3], which converts phosphatidylinositol 4-5 biphosphate (PIP2) to phosphatidylinositol 3-4-5 triphosphate (PIP3). Consequently, it activates multiple down-stream signaling pathways involved in cellular growth, motility, apoptosis and differentiation $[4,5]$. In contrast, this 
activity is regulated by the phosphate and tensin homolog (PTEN) which converts back PIP3 into PIP2. Hence a mutation that makes a hyperactive PIK3CA or an underactive PTEN, will end to overstimulate the PI3K/AKT/mTOR pathway leading to an oncogenic behavior $[5,6]$.

Since its discovery in 2004 [2], researchers have had a special attention to PIK3CA mutations as they considered them to have a potential use as prognostic and predictive factors. In addition, several authors have reported a significant association among PIK3CA mutations and positive prognostic factors [7-9], as well as a prolonged relapse free survival [10]. Nonetheless, this data is still controversial [11] as other authors have suggested that PIK3CA mutation are for the contrary associated with a negative prognosis $(6,10)$ and current therapy resistance (hormone and anti-HER2 therapy) $[12,13]$.

Traditionally, gene analysis in tumor samples have been conducted by Sanger sequencing, despite of its limitations [14-16]. Nevertheless, new technologies have emerged promising a better performance, enabling a quantitative and more sensitive gene analysis.

One of these technologies is the "digital Polymerase Chain Reaction" (dPCR) technology, which performs an absolute quantification of specific nucleic acid sequences by first dividing a sample in several individual partitions in chambers or droplets (depending on the platform), so at least one target molecule is contained in a partition. Then it performs simultaneous endpoint PCRs and count in a binary style ( 0 negative and 1 positive) the partitions where a fluorescence signal has been produced with a posterior Poisson analysis [17-19]. In contrast with other PCR techniques such as qPCR, dPCR does not require standard curves calibration and due to partition, it enhance the effective concentration of the objective nucleic acids and decreases the background effect of abundant molecules over rare or low frequent targets [20]. Thus, it is capable to detect until a theoretical $0.1 \%$ mutant allele frequency. Consequently, this technique has a great potential in personalized medicine as well as in cancer research [21].

There are several studies that have already used dPCR technology for mutation analysis with optimistic results [22-25]. Even though, most of them have commonly used Droplet Digital PCR (ddPCR), which is a water-oil droplet based platform that fractionate the sample in several droplet reactions [25], there is a novel chip-based platform, QuantStudio 3D Digital PCR System, which offers a highly precise and sensitivity absolute quantification with a similar quantity of partitions as ddPCR but with a simpler workflow [19] and a relatively low cost. However, there are still few studies using this new technique. Therefore, we designed a cross-sectional study, to determine the sensitivity of QuantStudio 3D Digital PCR system to detect PIK3CA mutations in breast cancer patients, in comparison with Sanger sequencing, as well as an analysis between patient clinicpathological features and PIK3CA mutational status detected by dPCR.

\section{Materials and Methods}

\subsection{Study Population}

Tumor/biopsy samples were obtained from patients with breast cancer diagnosed at the Instituto Nacional de Enfermedades Neoplásicas (INEN) in Lima-Peru and analyzed in a core Laboratory (Centro de Genética y Biología Molecular) at Universidad de San Martin de Porres. Patients were enrolled prospectively in the study between April and August of 2017 after signing the proper in-formed consent. The study protocol and informed consent were approved by Universidad de San Martín de Porres IRB (IRB00003251-FWA0015320) and the Protocols Review Committee from INEN. Biopsy samples were obtained when patient's tumor was not available. Additional information regarding demographic and clinicopathological information was obtained from clinical records. Breast cancer phenotypes were classified based on the St. Gallen International Expert Consensus from 2011 [26].

\subsection{Sample Processing and DNA Extraction}


For each case, hematoxylin and eosin-stained slides from tumor/biopsy FFPE blocks were reviewed by a pathologist to confirm and delimitate the area with neoplastic cells. These delimitated areas were then localized in the FFPE blocks and cut in eight slices of $4 \mathrm{~mm}$ thick. Genomic DNA extraction was performed according to the GeneJet FFPE DNA purification Kit (ThermoFisher Scientific, Boston, MA, USA) protocol. Tumor DNA was eluted in $80 \mu \mathrm{L}$ of the given elution buffer and stored at $-20^{\circ} \mathrm{C}$. Concentration and purity of DNA were determined using NanoDropTM Lite Spectrophotometer (ThermoFisher Scientific, Boston, MA, USA). The median time between the FFPE processing and DNA extraction was 112 days.

\subsection{PIK3CA Mutations Analysis}

Three mutations in the PIK3CA gene (E545K, H1047R and H1047L) were assessed using QuantStudio 3D Digital PCR System (ThermoFisher Scientific, Boston, MA, USA) and Sanger Sequencing. For digital PCR, $1.5 \mu \mathrm{L}$ of sample DNA was mixed with $0.75 \mu \mathrm{L}$ of 20x TaqMan Assay (Table S1), plus 7.5 $\mu \mathrm{L}$ of QuantStudio 3D Master Mix 2X and 5.25 $\mu \mathrm{L}$ of water. The total mixture of $15 \mu \mathrm{L}$ was then loaded in the QuantStudio 3D Digital PCR 20k Chips by the QuantStudioTM 3D Digital PCR Chip Loader. The Cycling conditions for exon 20 mutations (H1047R and H1047L) were an initial denaturation at $96^{\circ} \mathrm{C}$ for 10 minutes followed by 39 cycles of $60^{\circ} \mathrm{C}$ for two minutes, 30 seconds at $98^{\circ} \mathrm{C}$ and a final stage of two minutes at $60^{\circ} \mathrm{C}$ for extension. While for exon 9 mutation (E545K) were an initial denaturation at $96^{\circ} \mathrm{C}$ for 10 minutes followed by 40 cycles of $52^{\circ} \mathrm{C}$ for two minutes, 30 seconds at $98^{\circ} \mathrm{C}$ and a final stage of 10 minutes at $72^{\circ} \mathrm{C}$ for a final extension [27]. All samples were then left at $22^{\circ} \mathrm{C}$ for at least 20 minutes. Results were analyzed by QuantStudio 3D Analysis Suite ${ }^{\mathrm{TM}}$ Cloud Software (ThermoFisher Scientific, Boston, MA) based on the Poisson plus algorithm (v.4.4.10). The software calculated automatically the thresholds for FAM (mutant alleles) and VIC (wild type alleles) signals. However, to homogenize the results, reduce false positives and avoid observer bias we established a fixed threshold of 6000 relative fluorescence units for FAM on all the samples based on our most representative positive cases (Figure S1). Additionally, a quality threshold of 0.5 was established. Calibration process for $\mathrm{dPCR}$ can be found in supplementary material. Digital PCR assays were performed following the digital MIQE guidelines [28] (Figure S2, Table S11).

For Sanger sequencing, we used the ABI PRIMS 3500 (Applied Biosystems ${ }^{\mathrm{TM}}$, Foster City, CA, USA). Conventional PCR was carried on for exon 9 (E545K) and for exon 20 (H1047R and H1047L). Primers used and cycling conditions are presented in supplementary material (Table S2, S3 and S4). Before sequencing, samples were purified according to GeneJet PCR Purification Kit (ThermoFisher Scientific, Boston, MA, USA) protocol with the exception that we preheated the elution buffer at $60^{\circ} \mathrm{C}$ for at least 10 minutes to increase DNA concentration. DNA was then eluted in $35 \mu \mathrm{L}$ of the elution buffer supplied. Two independent readers analyzed the sequences and compared them with the reference sequence: NC_0000003.12 (GRCh38).

\subsection{Statistical Analysis}

To calculate the sensitivity, specificity, predictive positive value (PPV) and predictive negative value (PNV) of dPCR, we compared the 171 essays performed by dPCR against their respective results from Sanger sequencing. Each patient had three essays (one for each mutation) in both dPCR and Sanger sequencing. The analysis was performed with contingency tables from JavaStat (http://statpages.info/ctab2x2.html). Whereas, we used STATA v15.1 for the other statistical analysis. We estimate the Receptor Operator Characteristic (ROC) curve of dPCR against Sanger sequencing and calculate the area under the curve (AUC). The test qualification according the AUC curve might be from non-discriminatory (0.5 or minus) to outstanding (over 0.9) [29]. Additionally, we performed the chi- 
square test and Fisher exact test (when needed) to assess which clinicpathological feature were related to the mutational status of PIK3CA E545K and H1047R. Intra-rater analysis was estimated by Intraclass Correlation Coefficient (ICC) based on a single-rating ( $k=1)$, consistency of agreement, 2-way mixed-effects model. For all the analysis, we calculated $95 \%$ confident intervals (CI) and considered two-sided P value 0.05 as statistically significant threshold.

\section{Results}

\subsection{Patients Characteristics}

Sixty-nine patients accepted to participate in the study by signing the informed consent. However, one patient decided to quit and 11 were excluded because it was not possible to obtain their tumor and biopsy sample. A total of 57 patients were finally eligible for the study. Demographic and clin-ic-pathological characteristics are resume in Table 1. From them, 16 samples were primary tumor $(28.1 \%), 32(56.1 \%)$ residual tumors (had received neoadjuvancy), six were biopsies $(10.5 \%)$ and three $(5.3 \%)$ belonged to lymph nodes.

Table 1. Patient Demographic and Clinic-pathological Features $(\mathrm{N}=57)$

\begin{tabular}{|c|c|}
\hline Patient Feature & $\mathbf{N}(\%)$ \\
\hline \multicolumn{2}{|c|}{ Previous Neoadyuvancy } \\
\hline Yes & $38(66.7)$ \\
\hline No & $19(33.3)$ \\
\hline \multicolumn{2}{|l|}{ Age } \\
\hline$\leq 50$ & $22(38.6)$ \\
\hline$>50$ & $35(61.4)$ \\
\hline \multicolumn{2}{|l|}{ Stage } \\
\hline 0 & $1(1.8)$ \\
\hline I & $3(5.3)$ \\
\hline II & $10(17.5)$ \\
\hline III & $37(64.9)$ \\
\hline IV & $5(8.8)$ \\
\hline No evaluable & $1(1.8)$ \\
\hline \multicolumn{2}{|l|}{ Histological Type } \\
\hline Ductal & $46(80.7)$ \\
\hline Lobular & $4(7.0)$ \\
\hline Other & $7(12.3)$ \\
\hline \multicolumn{2}{|l|}{ HER-2 Receptor } \\
\hline Positive & $19(33.3)$ \\
\hline Negative & $34(59.7)$ \\
\hline No evaluable & $4(7.0)$ \\
\hline \multicolumn{2}{|l|}{ Estrogen Receptor } \\
\hline Positive & $37(64.9)$ \\
\hline Negative & $19(33.3)$ \\
\hline No evaluable & $1(1.8)$ \\
\hline \multicolumn{2}{|c|}{ Progesterone Receptor } \\
\hline Positive & $32(56.1)$ \\
\hline
\end{tabular}


Negative

No evaluable

Immunophenotype

Luminal A like

Luminal B like

HER-2 (+)

Basal-like

No evaluable

Lymph Node

Positive

Negative

\section{Systemic Metastasis}

Positive

Negative

\section{Hormonal Status}

Pre-menopause

Post-menopause

\section{Region of Birth}

Lima

Pacific coast

Andes

Amazon

Ki-67

$\geq 14$

$<14$

No evaluable
24 (42.1)

$1(1.8)$

9 (15.8)

28 (49.1)

10 (17.5)

$8(14.0)$

2 (3.5)

40 (70.2)

$17(29.8)$

52 (91.2)

13 (22.8)

44 (77.2)

19 (33.33)

20 (35.09)

13 (22.81)

5 (8.77)

41 (71.93)

10 (17.54)

6 (10.53)

Note: Percentage may not sum $100 \%$ due to rounding.

\subsection{PIK3CA Mutation Status}

Tumor DNA samples were analyzed by dPCR. Twenty-five patients (43.9\%) showed to have at least one mutation. Mean copies per partition, total volume of partition and partition number are shown in supplementary material (Table S5). From the three mutations, E545K was the most frequent with 18 cases (31.6\%), followed by H1047R 11 cases $(19.3 \%)$ and H1047L 3 cases (5.3\%). Examples of positive and negative cases can be seen in supplementary material (Figure S1). Furthermore, seven patients presented coexistence of two mutations, from which four had H1047R and E545K, and three H1047R and H1047L. On the other hand, regarding H1047R, H1047L and E545K mutations exclusively, Sanger sequencing identified six mutated cases (10.5\%) from which four (7\%) were E545K and two (3.5\%) H1047R. Neither H1047L nor mutation coexistence were identified by Sanger sequencing (Table 2). Dilution and intra-rater assay can be found in the supplementary material (Figure S2, Tables S6-S10). 
Table 2. PI3KCA Mutations detected by Digital PCR and Sanger sequencing

\begin{tabular}{|c|c|c|c|}
\hline Patient & Digital PCR & FAM Copies/ $\mu \mathrm{L}$ & Sanger sequencing \\
\hline 01 & E545K $(0.44 \%)$ & 2.93 & WT \\
\hline \multirow[t]{2}{*}{02} & H1047R (57.94\%) & 2364.8 & H1047R \\
\hline & E545K $(0.50 \%)$ & 10.75 & WT \\
\hline \multirow[t]{2}{*}{03} & H1047R (4.34\%) & 12.2 & WT \\
\hline & E545K (0.50\%) & 2.31 & WT \\
\hline 04 & WT & - & WT \\
\hline 05 & WT & - & WT \\
\hline 06 & WT & - & WT \\
\hline 07 & WT & - & WT \\
\hline 08 & WT & - & WT \\
\hline 09 & WT & - & WT \\
\hline 10 & WT & - & WT \\
\hline 11 & WT & - & WT \\
\hline \multirow[t]{2}{*}{12} & H1047R (0.35\%) & 3.1 & WT \\
\hline & E545K (0.89\%) & 10.14 & WT \\
\hline 13 & E545K (0.29\%) & 0.74 & WT \\
\hline 14 & E545K (11.66\%) & 229.0 & E545K \\
\hline \multirow[t]{2}{*}{15} & H1047R (0.24\%) & 1.6 & WT \\
\hline & E545K $(0.44 \%)$ & 2.13 & WT \\
\hline 16 & WT & - & WT \\
\hline 17 & $\mathrm{WT}$ & - & WT \\
\hline 18 & E545K (0.28\%) & 1.39 & WT \\
\hline 19 & E545K (54.60\%) & 3263.90 & E545K \\
\hline 20 & WT & - & WT \\
\hline 21 & $\mathrm{WT}$ & - & WT \\
\hline \multirow[t]{2}{*}{22} & H1047R (41.17\%) & 358.93 & H1047R \\
\hline & H1047L (0.30\%) & 1.57 & WT \\
\hline 23 & E545K $(0.25 \%)$ & 0.23 & WT \\
\hline 24 & E545K $(0.30 \%)$ & 7.01 & WT \\
\hline 25 & WT & - & WT \\
\hline 26 & WT & - & WT \\
\hline 27 & H1047R (14.11\%) & 216.90 & WT \\
\hline \multirow[t]{2}{*}{28} & H1047R (0.24\%) & 9.67 & WT \\
\hline & H1047L (0.85\%) & 4.79 & WT \\
\hline 29 & WT & - & WT \\
\hline 30 & WT & - & WT \\
\hline 31 & WT & - & WT \\
\hline 32 & WT & - & WT \\
\hline 33 & H1047R (12.48\%) & 6.5 & WT \\
\hline 34 & E545K (0.30\%) & 0.81 & WT \\
\hline 35 & WT & - & WT \\
\hline
\end{tabular}




\begin{tabular}{cccc}
36 & WT & - & WT \\
37 & H1047R $(9.64 \%)$ & 32.76 & WT \\
38 & WT & - & WT \\
39 & WT & - & WT \\
40 & E545K $(6.39 \%)$ & 85.81 & WT \\
41 & E545K $(2.25 \%)$ & 15.88 & WT \\
42 & WT & - & WT \\
43 & WT & - & WT \\
44 & WT & - & WT \\
45 & WT & - & WT \\
46 & WT & - & WT \\
47 & E545K $(34.85 \%)$ & 1567.30 & E545K \\
48 & E545K $(10.05 \%)$ & 53.96 & WT \\
49 & WT & - & WT \\
50 & WT & - & WT \\
51 & WT & - & WT \\
52 & E545K (17.66\%) & 4630.4 & E545K \\
53 & H1047R (11.42\%) & 76.06 & WT \\
54 & H1047R $(2.67 \%)$ & 17.61 & WT \\
& H1047L (5.13\%) & 36.63 & WT \\
55 & WT & - & WT \\
56 & WT & - & WT \\
57 & E545K $(0.24 \%)$ & 4.77 & WT \\
\hline
\end{tabular}

WT: Wild type.

\subsection{Sensitivity and Specificity Analysis}

For sensitivity and specificity analysis, Sanger sequencing was used as gold standard against dPCR. Digital PCR sensitivity showed to be $100 \%$ (95 CI 53-100\%), while the specificity $84.2 \%$ (95\% CI $83-84.2 \%$ ), PPV 18.8\% (95\% CI 9- 18\%) and PNV 100\% (95\% CI 98 $100 \%$ ). Furthermore, ROC analysis (Figure 1) showed an AUC of 0.998 (95\% CI 0.9781.00).

\subsection{PIK3CA Mutations and Patient's Clinic-pathological Features}

No statistical significance was found between clinic-pathological features and overall PI3KCA mutational status (Table 3). Additionally, mutational status was assessed individually by mutation (E545K and H1047R) showing a significant association between H1047R and Breast cancer Phenotype $(\mathrm{p}=0.019)$ and lymph node infiltration $(\mathrm{p}=0.046)$. Further, post hoc analysis through Pearson $\mathrm{X}^{2}$ test determined the association between H1047R and HER2 breast cancer phenotype (residuals 2.234, $\mathrm{p}=0.026$ ). 
Table 3. Association among PIK3CA mutations' and Clinic-pathological Features

\begin{tabular}{|c|c|c|c|c|c|c|c|c|c|c|}
\hline \multirow[t]{2}{*}{ CLINICAL FEATURES } & & $\mathrm{N}=57$ & E545K & \multirow[t]{2}{*}{$p$} & \multirow{2}{*}{$\begin{array}{c}\mathrm{N}=57 \\
(\%)\end{array}$} & \multirow{2}{*}{$\begin{array}{c}\text { H1047R } \\
\text { M=11 }\end{array}$} & \multirow[t]{2}{*}{$p$} & \multirow{2}{*}{$\begin{array}{c}\mathrm{N}=57 \\
(\%)\end{array}$} & \multirow{2}{*}{$\begin{array}{c}\text { ANY } \\
\text { Mutation } \\
\text { M=25 }\end{array}$} & \multirow[t]{2}{*}{$p$} \\
\hline & & $(\%)$ & $\mathbf{M}=18$ & & & & & & & \\
\hline Age (years) & $>50$ & $24(61.54)$ & $11(61.11)$ & & $29(63.04)$ & $6(54.55)$ & & $21(65.63)$ & $14(56)$ & \\
\hline \multirow[t]{4}{*}{ Stage } & In situ & $1(2.63)$ & $0(0)$ & 0.823 & $1(2.22)$ & $0(0)$ & 0.638 & $1(3.23)$ & $0(0)$ & 0.677 \\
\hline & I & $3(7.89)$ & $0(0)$ & & $2(4.44)$ & $1(9.09)$ & & $2(6.45)$ & $1(4)$ & \\
\hline & III & $25(65.79)$ & $12(66.67)$ & & $31(68.89)$ & $6(54.55)$ & & $22(70.97)$ & $15(60)$ & \\
\hline & IV & $3(7.89)$ & $2(11.11)$ & & $4(8.89)$ & $1(9.09)$ & & $2(6.45)$ & $3(12)$ & \\
\hline \multirow[t]{3}{*}{ Histological type } & Ductal & $30(76.92)$ & $16(88.89)$ & 0.428 & $39(84.78)$ & $7(63.64)$ & 0.124 & $27(84.38)$ & $19(76)$ & 0.695 \\
\hline & Lobular & $4(10.26)$ & $0(0)$ & & $2(4.35)$ & $2(18.18)$ & & $2(6.25)$ & $2(8)$ & \\
\hline & Others & $5(12.82)$ & $2(11.11)$ & & $5(10.87)$ & $2(18.18)$ & & $3(9.38)$ & $4(16)$ & \\
\hline Region & Amazon & $4(10.26)$ & $1(5.56)$ & & $3(6.52)$ & $2(18.18)$ & & $2(6.25)$ & $3(12)$ & \\
\hline \multirow[t]{5}{*}{ Immunophenotype } & Basal-like & $5(13.51)$ & $3(16.67)$ & 0.622 & $8(17.39)$ & $0(0)$ & 0.019 & $5(15.63)$ & $3(13.04)$ & 0.307 \\
\hline & Luminal A & $5(13.51)$ & $4(22.22)$ & & $6(13.04)$ & $3(33.33)$ & & $3(9.38)$ & $6(26.09)$ & \\
\hline & Luminal B & $21(56.76)$ & $7(38.89)$ & & $26(56.52)$ & $2(22.22)$ & & $19(59.38)$ & $9(39.13)$ & \\
\hline & HER-2 + & $6(16.22)$ & $4(22.22)$ & & $6(13.04)$ & $4(44.44)^{*}$ & & $5(15.63)$ & $5(21.74)$ & \\
\hline & No eval. & . & & & & . & & & & \\
\hline \multirow[t]{2}{*}{ HER-2 } & Positive & $13(37.14)$ & $6(33.33)$ & 0.784 & $14(31.82)$ & $5(55.56)$ & 0.255 & $11(36.67)$ & $8(34.78)$ & 0.887 \\
\hline & Negative & $22(62.86)$ & $12(66.67)$ & & $30(68.18)$ & $4(44.44)$ & & $19(63.33)$ & $15(65.22)$ & \\
\hline
\end{tabular}




\begin{tabular}{|c|c|c|c|c|c|c|c|c|c|c|}
\hline Estrogen & Positive & $26(68.42)$ & $11(61.11)$ & 0.589 & 31 (67.39) & $6(60)$ & 0.720 & $21(65.63)$ & $16(66.67)$ & 0.935 \\
\hline receptor & Negative & $12(31.58)$ & $7(38.89)$ & & $15(32.61)$ & $4(40)$ & & $11(34.38)$ & $8(33.33)$ & \\
\hline \multirow[t]{2}{*}{ Progesterone receptor } & Positive & $24(63.16)$ & $8(44.44)$ & 0.186 & $27(58.7)$ & $5(50)$ & 0.615 & $19(59.38)$ & $13(54.17)$ & 0.697 \\
\hline & Negative & $14(36.84)$ & $10(55.56)$ & & $19(41.4)$ & $5(50)$ & & $13(40.63)$ & $11(45.83)$ & \\
\hline \multirow{2}{*}{$\begin{array}{l}\text { Lymph nodes } \\
\text { infiltration }\end{array}$} & Positive & $27(69.23)$ & $13(72.22)$ & 0.819 & 35 (76.09) & $5(45.45)$ & 0.046 & $24(75)$ & $16(64)$ & 0.368 \\
\hline & Negative & $12(30.77)$ & $5(27.78)$ & & $11(23.91)$ & $6(54.55)$ & & $8(25)$ & $9(36)$ & \\
\hline Ki-67 & $>=14$ & $29(85.29)$ & $12(70.59)$ & & $35(83.33)$ & $6(66.67)$ & & $26(89.66)$ & $15(68.18)$ & \\
\hline Systemic & Positive & $3(7.69)$ & $2(11.11)$ & 0.646 & $4(8.70)$ & $1(9.09)$ & 1.000 & $2(6.25)$ & $3(12)$ & 0.645 \\
\hline metastasis & Negative & $36(92.31)$ & $16(88.89)$ & & 42 (91.30) & $10(90.91)$ & & 30 (93.75) & $22(88)$ & \\
\hline Menstrual & Pre-menopause & $10(25.64)$ & $3(16.67)$ & 0.520 & $11(23.91)$ & $2(18.18)$ & 1.000 & $8(25)$ & $5(20)$ & 0.655 \\
\hline
\end{tabular}

The analysis was performed with the Chi-square or Fisher's exact test. The numbers highlighted in bold indicate significant differences $(p<0.05)$.

M: mutations. * Significantly associated under $X^{2}$ residual evaluation. 


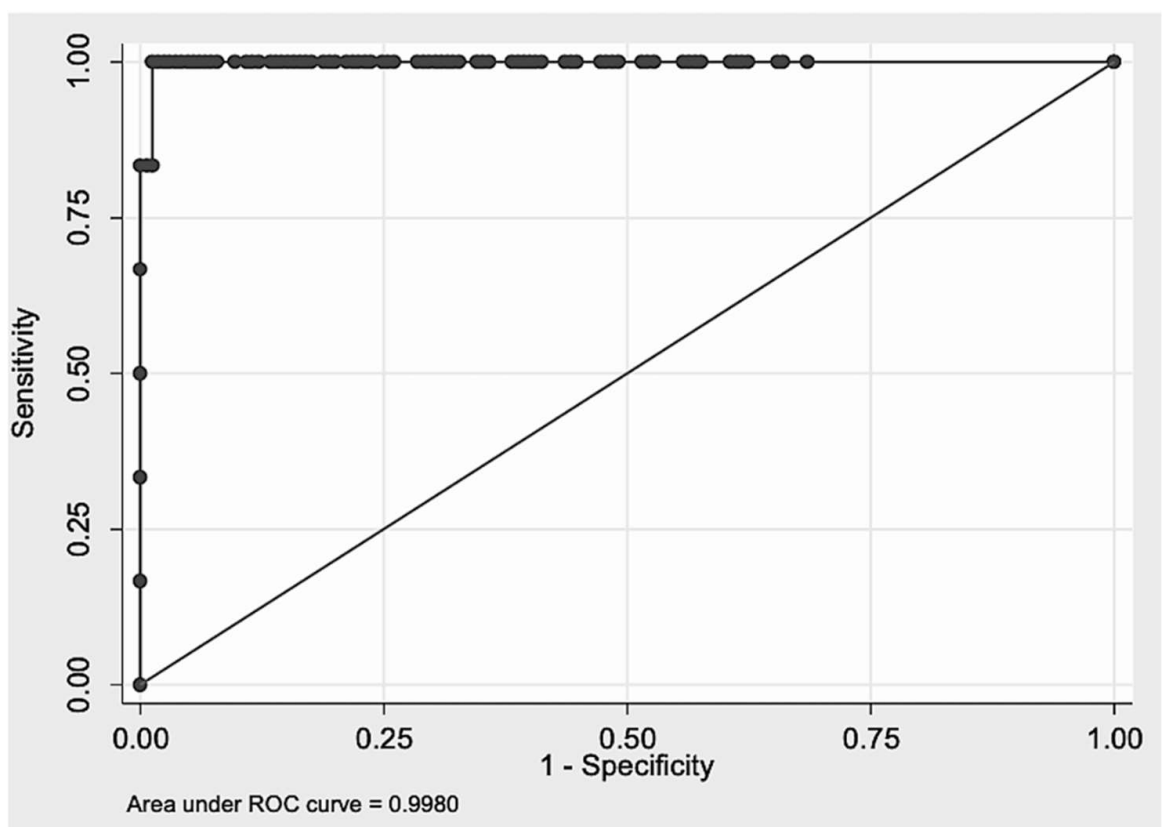

Figure 1. Receiver operator characteristics (ROC) curve for PIK3CA mutations detected by dPCR.

\section{Discussion}

Digital PCR is a relatively new technique that enable mutation analysis of low rate target molecules due to its partition properties and Poisson analysis [20]. In this study we compared the QuantStudio 3D digital PCR system against Sanger sequencing, as gold standard, to assess its sensitivity, specificity and overall performance in the detection of three PIK3CA mutations in breast cancer tumors. In our study, dPCR revealed a prevalence of $43.9 \%$ of three PIK3CA mutations exclusively (E545K, H1047R and H1047L). Even though, our results are concordant with the one reported by Beaver et al. in a similar platform (ddPCR) [30], it differs in almost $8 \%$ with the overall PI3KCA mutations prevalence in breast cancer estimated by Whole Genome Sequencing (WGS) and Whole Exome Sequencing (WES) studies reported in TGCA database (36\%) [1]. However, this incon-sistency can be explained by the difference on sensitivity between APCR and TGCA techniques. Furthermore, Sanger sequencing identified only $10.5 \%$ of mutated tumors, which is low compared with dPCR, but similar to previous studies taking in consideration only E545K, H1047R and H1047L mutations [31,32].

In terms of performance, sensitivity and specificity, dPCR showed excellent results as compared to Sanger sequencing. Nevertheless, dPCR sensitivity had a broad CI which range from 53 to $100 \%$ and a low PPV. These might be explained by the 19 cases that Sanger sequencing was not able to detect as previous studies have reported a detection limit of $15-30 \%$ for Sanger sequencing $[15,16]$. In our study, Sanger sequencing was able to detect until a mutation rate of $11.66 \%$ according to dPCR. However, it draws attention cases 34 and 42 which were not detected by Sanger sequencing in spite of a mutation rate for H1047R of $14.11 \%$ and $12.48 \%$ respectively by dPCR. This issue can be due to the number of copies per $\mu \mathrm{L}$ in the sample as case 18 , with a mutation rate of $11.66 \%$ for E545K, showed to have 229 mutant copies/ $\mu \mathrm{L}$, compared to 216 and 6.5 copies/ $\mu \mathrm{L}$ from patient 34 and 42 respectively; suggesting the importance of not only the proportion of mutant alleles over the wild types but also the overall number of copies within the sample for Sanger sequencing analysis. 
Individually, E545K was the most frequent mutation, followed by H1047R and H1047L in both techniques which contrast with previous data, where H1047R mutation represent approximately $50 \%$ of PIK3CA total mutation rate and E545K up to $20 \%$ [33]. Nevertheless, it is important to note that most of E545K cases detected by dPCR have a mutation rate lower than $1 \%$, which might have been underestimated in previous studies $[8,31,32,34]$. Additionally, dPCR detected seven patients with mutation coexistence, not identified by Sanger sequencing. This phenomenon has been reported previously $[30,35,36]$ and may reflects the intra-heterogeneous characteristics from breast cancer tumors and has been suggested to have an important role in tumor progression and treatment [37]. Further, it is suggested that therapy failures and metastasis may be due to the outgrowth of resistant sub-clones present before the beginning of the treatment and underestimated by standard techniques as shown by recent studies [38,39]. Because of this, resembles the importance on developing more sensitive techniques dPCR capable to detect potential resistant sub-clones and guaranty a personalized medicine.

Furthermore, we found a significant association of HER2 phenotype and lymph nodes infiltration with H1407R mutation. Event though, previous studies haven't reported a correlation or association between this mutation and HER2 phenotype, its presence is associated with a worse overall survival in breast cancer patients and therapy resistance $[40,41]$. Besides, animal studies have shown an acceleration on tumor progression as well as an enhancement on metastasis potential in mice with tumors expressing both H1047R mutations and HER2 overexpression [42,43] suggesting a synergic effect. This same way may also explain the association with lymph node infiltration. However, this analysis was mainly exploratory and may lack of enough power. Further considerations should be taken into account in future studies.

\section{Conclusions}

Our study has shown a good performance of dPCR, compared with Sanger sequencing, in the detection of three PIK3CA mutations with high sensitivity and specificity values. Besides, due to the detection of low rate mutations and mutation co-existence that Sanger sequencing could not detect, dPCR has the potential to become an important tool for gene analysis and personalized medicine. Thus, future studies with larger populations should be performed to confirm and extent our results.

Supplementary Materials: The following are available online at www.mdpi.com/xxx/s1, Figure S1: Examples of positive and negative samples for each analyzed mutations, Figure S2: Three examples from the dilution assays' samples, Table S1: Catalog number of QuantStudio TM 3D Digital PCR Products, Table S2: Primers used for conventional PCR. Table S3: Cycling conditions PIK3CA primers exon 20, Table S4: Cycling conditions PIK3CA primers exon 09, Table S5: Mean $\lambda$, partition volume, total partition number and total volume of part, Table S6: PIK3CA H1047R mutations dilution assay by dPCR, Table S7: PIK3CA H1047L mutations dilution assay by dPCR, Table S8: PIK3CA E545K mutations dilution assay by dPCR, Table S9: PIK3CA mutations dilution assay by dPCR, Table S10: Inter-rater assay results from mutant allele frequency, Table S11: digital MIQE checklist for authors, reviewers and editors.

Author Contributions: Conceptualization, J.B, O.A.C, A.A., and S.G.L.; Methodology, J.M.C., H.G.M., A.M.C., J.B., O.A.C., P.D., J.P., J.A. and S.G.L.; formal analysis, O.A.C., S.G.L., P.D.; investigation, and writing-original draft preparation, S.G.L., A.M.C., and R.F..; writing-review and editing, S.G.L, J.B, O.A.C, and R.F.; funding acquisition, J.B, O.A.C, and R.F. All authors have read and agreed to the published version of the manuscript.

Funding: This study was funded by research grants from the Facultad de Medicina Humana de la Universidad de San Martín de Porres (Project E1001-2016038), Programa Nacional de Innovación para la Competitividad y Productividad - Innóvate Perú (Grant Number 138-PNICP-PIAP-2015), Oncosalud-AUNA and Origenetica SAC. 
Institutional Review Board Statement: Patients were enrolled prospectively in the study between April and August of 2017 after signing the proper informed consent. The study protocol and informed consent were approved by Universidad de San Martín de Porres IRB (IRB00003251FWA0015320) and the Protocols Review Committee from INEN.

Informed Consent Statement: Informed consent was obtained from all subjects involved in the study.

Conflicts of Interest: The authors declare no conflict of interest.

\section{References}

1. TGCA publication guidelines. Available online: cbioportal.org

2. Samuels Y, Wang Z, Bardelli A, Silliman N, Ptak J, Szabo S et al. High frecuency of mutations of the PIK3CA gene in human cancers. Science. 2004; 304(5670): 554.

3. Liu P, Cheng H, Roberts TM, Zhao JJ. Targeting the phosphoinositide 3-kinase (PI3K) pathway in cancer. Nat Rev Drug Discov. 2009; 8(8): 627-44.

4. Takeshita T, Yamamoto Y, Yamamoto-Ibusuki M, Inao T, Sueta A, Fujiwara S et al. Prognostic role of PIK3CA mutations of cell-free DNA in early-stage triple negative breast cáncer. Cancer Sci. 2015; 106(11): 1582-89.

5. Mankoo PK, Sukumar S, Karchin R. PIK3CA somatic mutations in breast cancer: Mechanistic insights from langenn dynamic simulation. Proteins. 2009 May 1; 75(2): 499-508.

6. Samuels Y, Diaz LA Jr, Schmidt-Kittler O, Cummins JM, Delong L, Cheong I et al. Mutant PIK3CA promotes cell growth and invasion of human cancer cells. Cancer Cell. 2005 June; 7(6):561-73.

7. García-Murillas I, Pearson A, Lambros M, Natrajan R, Segal C, Dowsett M, et al. Analysis of PIK3CA mutation abundance in primary breast cancer with droplet digital PCR identifies frequent sub-clonal PIK3CA mutations in ER negative and/ or HER2 positive breast cancer. Cancer Res 2013;73(24 Suppl): Abstract nr P2-08-01.

8. Cizkova M, Susini A, Vacher S, Cizeron-Clairac G, Andrieu C, Driouch K et al. PIK3CA mutation impact on survival in breast cancer patients and in ER. Breast Cancer Research. 2012; 14(1). R28.

9. Pérez-Tenorio G, Alkhori L, Olsson B, Ahnström Waltersson M, Nordenskjöld B, Rutqvist LE et al. PIK3CA Mutations and PTEN Loss Correlate with Similar Prognostic Factors and Are Not Mutually Exclusive in Breast Cancer. Clin Cancer Res. 2007; 13(12): 3577-84.

10. Pang B, Cheng S, Sun SP, An C, Liu ZY, Feng X et al. Prognostic role of PIK3CA mutations and their association with hormone receptor expression in breast cancer: a meta-analysis. Sci Rep. 2014: 4; 6255.

11. Samuels Y, Waldman T. Oncogenic mutations of PIK3CA in human cancers. Curr Top Microbiol Immunol. 2010;347:21-41.

12. Mayer IA, Arteaga CL. The PI3K/AKT Pathway as a Target for Cancer Treatment. Annu Rev Med. 2016;67:11-28.

13. Paplomata E, O'Reagan R. The PI3K/AKT/Mtor pathway in breast cancer: targets, trials and biomarkers. Ther Adv Med Oncol. 2014 Jul; 6(4): 154-166.

14. Kircher M and Kelso J. High-throughput DNA sequencing-concepts and limitations. Bioessays.2010;32(6):524-36. doi: 10.1002/bies.200900181.

15. Tsiatis AC, Norris-Kirby A, Rich RG, Hafez MJ, Gocke CD, Eshleman JR et al. Comparison of Sanger Sequencing, Pyrosequencing, and Melting Curve Analysis for Detection of KRAS Mutations: Diagnostic and Clinical Implications. J Mol Diagn. 2010 Jul;12(4):425-32.

16. Yunk TK, Chan KC, Mok TS, Tong J, To KF, Lo YM. Single-molecule detection of epidermal growth factor receptor mutations in plasma by microfluidics digital PCR in non-small cell lung cancer patients. Clin Cancer Res. 2009 Mar 15;15(6):207684.

17. Luthra R, Singh RR, Patel KP, editors. Clinical Applications of PCR. 3era ed. Nueva York: Humana Press; 2016.

18. Oshiro C, Kagara N, Naoi Y, Shimoda M, Shimomura A, Maruyama N et al. PIK3CA mutations in serum DNA are predictive of recurrence in primary breast cáncer patients. Breast Cancer Res Treat. 2015; 150(2):299- 307.

19. Thermo Fisher Scientific [sede web]. Thermo Fisher Scientific; 2018 [acceso Marzo 07 de 2018].QuantStudio 3D Digital PCR System. Disponible en: https://www.thermofisher.com/pe/en/home/life-science/pcr/digital-pcr/quantstudio-3d-digitalpcr-system.html

20. Basu AS. Digital Assays Part I: Partioning Statistics and Digital PCR. SLAS Technology. 2017; 22(4):369-86. doi: $10.1177 / 2472630317705680$.

21. Y. K. Wong, H. F. Tsang, V. W. Xue, C. M. Chan, T. C. Au, W. C. Cho, L. W. Chan \& S. C. Wong (2017) Applications of digital PCR in precision medicine, Expert Review of Precision Medicine and Drug Development, 2:3, 177-186, DOI: 10.1080/23808993.2017.1347482

22. Denis JA, Patroni A, Guillerm E, Pèpin D, Benali-Furet N, Wechsler J et al. Droplet digital PCR of circulating tumor cells from colorectal cancer patients can predict KRAS mutations before surgery. Mol Oncol. 2016 Oct;10(8):1221-31

23. Earl J, Garcia-Nieto S, Martinez-Avila JC, Montans J, Sanjuanbenito A, Rodriguez-Garrote M et al. Circulating tumor cells (Ctc) and kras mutant circulating free Dna (cfdna) detection in peripheral blood as biomarkers in patients diagnosed with exocrine pancreatic cancer. BMC Cancer. 2015 Oct 24;15:797. 
24. Gu J, Zang W, Liu B, Li L, Huang L, Li s et al. Evaluation of digital PCR for detecting low-level EGFR mutations in advanced lung adenocarcinoma patients: a cross-platform comparison study. Oncotarget. 2017 Sep 15; 8(40): 67810-67820.

25. Otsuji K, Sasaki T, Tanaka A, Kunita A, Ikemura M, Matsusaka K et al. Use of droplet digital PCR for quantitative and automatic analysis of the HER2 status in breast cancer patients. Breast Cancer Res Treat. 2017 Feb;162(1):11-18.

26. Kondov B, Milenkovikj Z, Kondov G, Petrushevka G, Basheska N, Bogdanovska-Todorovska M et al. Presentation of the Molecular Subtypes of Breast Cancer Detected by Immunohistochemistry in Surgically Treated Patients. Open Access Maced J Med Sci. 2018; 6(6):961-67. doi: 10.3889/oamjms.2018.231.

27. García-Saenz JA, Ayllón P, Laig M, Acosta-Eyzaguirre D, García-Esquinas M, Montes Myriam, et al. Tumor burden monitoring using cell-free tumor DNA could be limited by tumor heterogeneity in advanced breast cancer and should be evaluated together with radiographic imaging. BMC Cancer. 2017;17:210.

28. Hugget JF, Foy Ca, Benes V, Emslie K, Garson JA, Haynes R et al. The digital MIQE guidelines: Minimum Information for Publication of Quantitative Digital PCR Experiments. Clin Chem. 2013; 59(6):892-902. doi: 10.1373/clinchem.2013.206375.

29. Mandrekar JN. Receiver Operating Characterisitic Curve in Diagnostic Test Assesment. J Thorac Oncol. 2010; 5(9): 1315-16.

30. Beaver JA, Jelovac D, Balukrishna S, Cochran RL, Croessmann S, Zabransky DJ et al. Detection of Cancer DNA in Plasma of Patients with Early-Stage Breast Cancer. CLIN CANCER RES. 2014; 20(10): 2631-42.

31. Castaneda CA, Lopez-Ilasaca M, Pinto JA, Chirinos-Arias M, Doimi F, Neciosup SP et al. PIK3CA mutations in Peruvian patients with HER2-amplified and triple negative non-metastatic breast cancers. Hematol Oncol Stem Cell Ther. 2014; 7(4): $142-48$.

32. Liedke C, Cardone L, Tordai A, Yan K, Gomez HL, Barajas Figueroa LJ et al. PIK3CA-activating mutations and chemotherapy sensitivity in stage II-III breast cancer. Breast Cancer Res. 2008; 10(2). doi: 10.1186/bcr1984

33. My Cancer Genome homepage. My Cancer Genome. http://www.mycancergenome.org [accessed February 1, 2019]

34. Jensen JD, Knoop A, Laenkholm AV, Grauslund M, Jensen MB, Santoni-Rugiu E et al. PIK3CA mutations, PTEN, and pHER2 expression and impact on outcome in HER-2 positive early-stage breast cancer patients treated with adjuvant chemotherapy and trstuzumab. Ann Oncol. 2012; 23(8):2034-42. doi.10.1093/annonc/mdr546.

35. Higgins MJ, Jelovac D, Barnathan E, Blair B, Slater S, Power P et al. Detection of Tumor PIK3CA Status in Metastatic Breast Cancer Using Peripheral Blood. Cain Cancer Res. 2012; 18(12): 3462-3469.

36. Stemke-Hale K, Gonzales-Angulo AM, Lluch A, Neve RM, Kuo WL, Davies M et al. An Integrative Genomic and Proteomic Analysis of PIK3CA, PTEN, and AKT Mutations in Breast Cancer. Cancer Res. 2008; 68(15): 6084-91.

37. Martelotto LG, Ng CKY, Piscuoglio S, Weigelt B and Reis-Filho JS. Breast cancer intra-tumor heterogeneity. Breast Cancer Res. 2014; 16(3):210. doi: 10.1186/bcr3658

38. Janiszewska M, Liu L, Almendro V, Kuang Y, Paweletz C, Sakr RA et al. In situ single cell analysis identifies heterogeneity for PIK3CA mutation and HER2 amplification in HER2+ breast cancer. Nat Genet. 2015; 47(10):1212-19. doi: 10.1038/ng.3391

39. Yates LR, Gerstung M, Knappskog S, Desmedt C, Gundem G, Van Loo P, et al. Subclonal diversification of primary breast cancer revealed by multiregion sequencing. Nat Med. 2015; 21(7): 751-9. doi: 10.1038/nm.3886

40. Jensen JD, Knoop A, Laenkholm AV, Grauslund M, Jensen MB, Santoni-Rugiu E et al. PIK3CA mutations, PTEN, and pHER2 expression and impact on outcome in HER2-positive early-stage breast cancer patients treated with adjuvant chemotherapy and trastuzumab. Ann Oncol. 2012;23(8):2034-42. doi: 10.1093/annonc/mdr546.

41. Mangone FR, Bobrovnitchaia IG, Salaorni S, Manuli E, Nagai MA. PIK3CA exon 20 mutations are associated with poor prognosis in breast cancer patients. Clinics (Sao Paulo). 2012;67(11):1285-90.

42. Cheng H, Liu P, Ohlson C, Xu E, Symonds L, Isabella A, et al. PIK3CA(H1047R)- and Her2-initiated mammary tumors escape PI3K dependency by compensatory activation of MEK-ERK signaling. Oncogene. 2016;35(23):2961-70. doi: 10.1038/onc.2015.377.

43. Hanker AB, Pfefferle AD, Balko JM, Kuba MG, Young CD, Sánchez V et al. Mutant PIK3CA accelerates HER2-driven transgenic mammary tumors and induces resistance to combinations of anti-HER2 therapies. Proc Natl Acad Sci U S A. 2013;110(35):1437-7. doi: 10.1073/pnas.1303204110. 